\title{
The research of the pulse oxygen supply based on the fuzzy control technology
}

\author{
Faling $\mathrm{Hu}^{1,2, *}$, Tongfeng $\mathrm{Niu}^{1,2}$, Jun $\mathrm{Yao}^{1,2}$, Bingyan $\mathrm{Cui}^{2}$, Haoxing $\mathrm{Xu}^{1,2}$, Hong Liu ${ }^{1,2}$, \\ Shujuan Chen ${ }^{1,2}$, and Yusong Zhou ${ }^{1,2}$ \\ ${ }^{1}$ Laboratory of emergency Safety and Rescue Technology, Hubei 441003, China \\ ${ }^{2}$ Hubei Institute of Aerospace Chemical Technology, Hubei 441003, China
}

\begin{abstract}
According to the working principle of pulse oxygen supplies, we analyze how to realize the control of the oxygen flow by double different valves. a two-dimensional fuzzy control system is proposed to solve the unstable problem, which is brought by the shortcomings of the open-loop control system. We add a new parameter, the rate of the differential pressure signal changes, which contributes to a close -loop control system and increases the stability of the system. The experiments and the data show that the fuzzy control system make the process of breathing much more comfortable and solve the hysteresis and overshoot caused by the open-loop control system. The product reliability has been greatly improved.
\end{abstract}

\section{Introduction}

The normal flight height of the new generation military helicopter is $(2000 \sim 4000) \mathrm{m}$, with the limit of $(5000 \sim 6000) \mathrm{m}$. This kind of helicopter is normally non-closed cockpit and not equipped with the oxygen supply system. Because of the reduction of atmospheric pressure in the high, the oxygen partial pressure becomes lower and lower. As a result, the pilots get less oxygen from the inhaled gas, which will directly lead to hypoxia symptoms when they fly in the high. This is an important factor that makes a great difference on the working efficiency and life safety in the high flight environment[1].

At present, helicopters are gradually equipped with the oxygen supply system in the United States and some other developed countries. US PHODS has developed a single pulse oxygen supply device and a double pulse oxygen supply device which makes the maximum utilization use of oxygen and achieve a breakthrough in the design of its weight and size; India IAF has developed an oxygen supply system which can be working for more than two hours. This system was initially developed for the "Cheetah" helicopter, and their next plan is to develop a gas supply system which can be equipped for rice-17 and Dhruv helicopters. However, the field of technology in china is not mature enough. In spite of the huge amount, Chinese active helicopter shave not yet installed an oxygen supply system so far.

\footnotetext{
* Corresponding author: naught580@126.com
} 
As the key control unit of the oxygen supply system, oxygen regulator can follow the pilot's breathing pace to regulate the oxygen supply, to ensure the pilot's essential physiological needs[2]. According to the design of foreign helicopter oxygen supply system, the electronic oxygen regulator has a great advantage on smaller size, lighter weight, more flexible adjustment, easier maintenance and use[3]. Especially when the oxygen system works in a low output pressure, the regulator still keeps the regulation to the oxygen flow, which is a significant advantage.

The pulse oxygen supply system is designed specifically for the helicopter flight environment. It provides the pilot an extra supplement for the oxygen. The exploration of the control rules and the design of the controller itself occupy a dominant position in the system. Especially in high altitude flight above $3000 \mathrm{~m}$, the opening point and the last time of the valve determines the performance of the oxygen supply system. Whether the electronic oxygen regulator can adjust the flow rate with height changes automatically affects the system to achieve timely, precise and large amount of air supplement[4].

\section{The principle of the electronic pulse oxygen regulator}

The electronic oxygen regulator described in this paper has three kinds of working mode with two different electronic valves. It can make a maximum efficiency use of oxygen at high altitude.

As shown in Figure 1, the inlet tube is supplied with high-pressure pure oxygen, and the outlet tube is connected to the breathing cannula. The two electronic valves adjust the oxygen supplement according to the flight height and the breathing frequency. The differential pressure sensor detects the pressure signal changes between the outlet tube and the outside air environment. The circuit board controls the opening and closing state of the two electronic valves based on the height changes. When the pilot inhales, the outlet pressure is less than the outside air environment, then the circuit board control the electronic valves open, oxygen supplied to the pilots, until the pressure changes between the outlet tube and the outside air environment is offset or change to nearly zero, the valves are controlled to close.

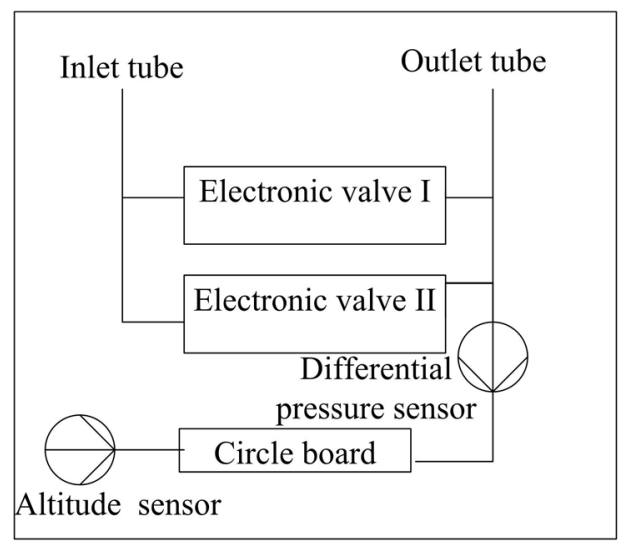

Fig. 1. The electronic oxygen regulator Schematic diagram.

According to the Figure 1, we can get a conclusion that the state of the valve controls the intake state of the oxygen source and is closely related to the pilot's breathing state. Different pilots show different physical fitness and their breathing characteristics are different too. As a result, their needs for oxygen supply are of course not the same. Therefore, only to find out the best control rules of the valves' opening and closing, can we 
make a comfortable breathing supplement for the pilots and achieve an efficient use of limited oxygen source.

\section{The regular pattern of breathing}

The regular pattern of breathing is shown in Figure 2, which expresses the curve of the outlet tube pressure versus respiration as time goes without any external sources.

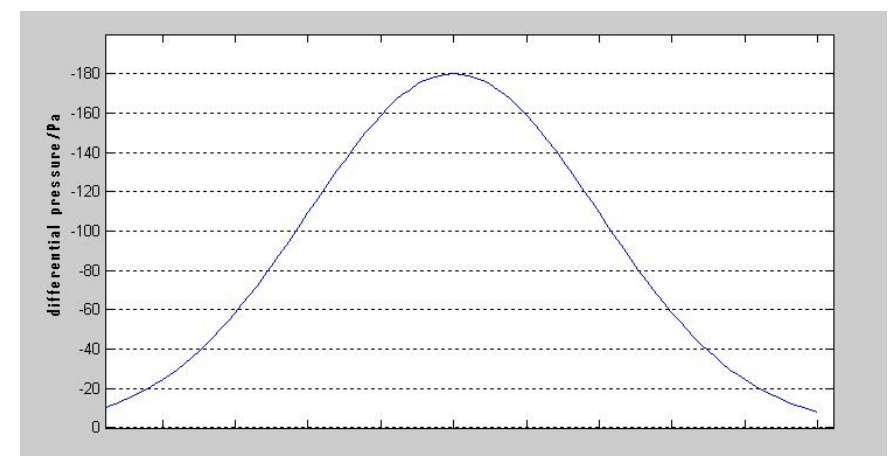

Fig.2. The curve of the breathing regular pattern.

At the beginning of the inhalation, the electronic valves keep the closed state. Once the differential pressure sensor detects the pressure changes over $20 \mathrm{~Pa}$ between the outlet tube and the outside air environment, the controller controls the electronic valves to change their state to be opening. Then the oxygen enters the outlet tube, passing through the nasal cannula, applied to the pilots. Entering oxygen lead to the pressure increase of the outlet tube and offset the differential pressure signal of course. As a result, the differential pressure signal gradually approaches to $0 \mathrm{~Pa}$. As the inhalation process goes on, the valves continue the opened state to keep the pressure balance of the outlet tube.

When the pilot exhales air outside, they sent some air to the outlet tube, which contribute to the pressure increase of the outlet tube. Once the differential pressure sensor detects the increases over $10 \mathrm{~Pa}$, the controller controls the electronic valves to change their state to be closed. Therefore, a delay will be brought when the controller tries to close the valves at the exhalation cycle, reducing the oxygen utilization seriously.

Based on the analysis above and the respiratory curve in Figure 2, the following control laws can be summed up: according to the differential pressure signal changes, the controller adjusts the electronic valves to be opened. After the valves opened, the oxygen is supplied in the form of pulse to the pilot, which means the valves are opened for a period of time. As for how long this period of time last, it bases on the flight altitude. Then the pressure of the outlet tube will get complemented and the differential pressure approach to $0 \mathrm{~Pa}$.As a result, a stable balance is rebuilt. Once the opening time is controlled, it's not necessary to detect the differential pressure signal changes to adjust the valves closing, the phenomenon of delay can be eliminated in the end.

\section{Research on the Control Method of double Valves}

The control principle of the electronic pulse oxygen regulator can be expressed in block diagram, as shown in Figure 3. 


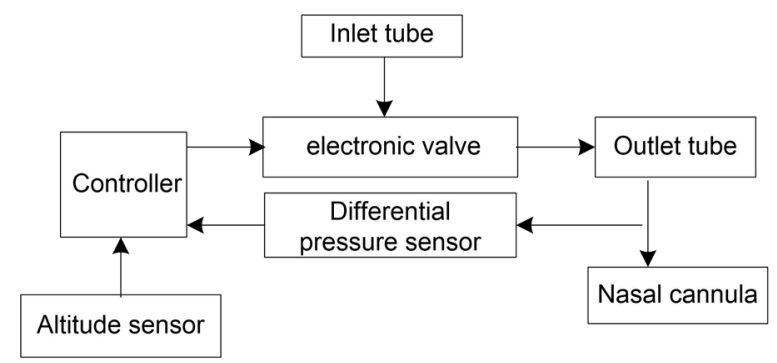

Fig. 3. The control schematic diagram of an electronic pulse oxygen regulator.

When the pilot tries to get the oxygen supplied through the nasal cannula during the first phase of inhalation, the pressure of the outlet tube gets down. The controller gets the message from the differential pressure sensor and then controls the valve to turn on in accordance with a certain control method programmed in the controller chip. Supply oxygen passes through the nasal cannula to the pilot. The outlet tube pressure changes with the oxygen gas entering into. We can get a conclusion that two factors cause a change of the differential pressure detected by the sensor. One is the pilot's breathing activity and the second is the oxygen gas entering into the outlet tube. In order to achieve the most comfortable breathing feeling, we must build a certain dynamic balance between the two factors. Otherwise, too small oxygen flow will affect the pilot's breathing comfort, even cause a hypoxia symptoms. Subjected to the limited oxygen source, too large oxygen flow will shorten the use of oxygen equipment. Therefore, controlling the opening and closing state of the electronic valves is the key to the design of the electronic pulse oxygen regulator.

\subsection{Control method of the double valves}

Breathing can be divided into two modes, calm breathing mode and strengthen breathing mode. Our usual breathing activity is part of calm breathing mode, in which a little breathing gas is needed. However, when we have an exercise or feel comfortable from hypoxia, more extra amount of breathing gas is needed of course and such is the so called strengthen breathing mode. The process of inhalation usually takes about $0.8 \mathrm{~s}$ while the exhalation takes $1.2 \mathrm{~s}$. Then the pause time is $1 \mathrm{~s}$ if we set the breathing frequency to be $20 \mathrm{bpm}$. As we all know, different pilot owns the unique inspiration curve, even the same pilot act a different breathing characteristics in different flight height. In another word, there isn't a completely same inspiration curve in the world. Despite all this, the trend of breathing movement is always consistent.

In the traditional control units, engineers would like to choose a stepping motor as the execution unit to achieve the continuous flow controlling. The control of a motor is so complicated and unstable that a mature product has never been available. On the other hand, we don't need such a precise flow controlling actually. Therefore, this paper selects two groups of electronic valves different in the size of flow path, which have formed three types of opening degree. This paper tries to come up with a fuzzy control method to choose the best type of opening degree(U) according to the pilot's breathing curve. As shown in Table 1 below:

Table 1. Truth table of electronic valves combination state.

\begin{tabular}{|c|c|c|c|c|}
\hline U & ZO & S & M & B \\
\hline Electronic valve I & 0 & 1 & 0 & 1 \\
\hline Electronic valve II & 0 & 0 & 1 & 1 \\
\hline
\end{tabular}


As shown in Table 1, the flow rate of the electronic valve I is smaller than that of the electronic valve II. From the state of $\mathrm{ZO}$ to $\mathrm{B}$, the valve opening degree increases in turn.

Z0 means double valves closed; $\mathrm{S}$ means small valve open; $\mathrm{M}$ means large valve open; B means double valves open.

Under different flight height conditions, the opening electronic valve will let the oxygen pass through and enter into the nasal cannula. with the depth of breathing activities, the monitor controller adjusts the valve's opening degree further to meet the pilot breathing needs, based on the signal detected by the differential pressure sensor. The greater the differential pressure is, the larger the opening degree is needed. Once the positive pressure occurs, namely the pressure of the outlet tube is over that of the outside air environment, $U$ $=\mathrm{ZO}$, the regulator turns off the oxygen supply automatically.

\subsection{Control methods based on fuzzy control rules}

Fuzzy control is a kind of control theory based on language rules and fuzzy derivation in modern control theories, and it is an important branch of intelligent control. As a simulation of human thinking, fuzzy control has been widely used in industrial control fields[5].

The control of the electronic valves is designed as a two-dimensional MISO fuzzy controller. The input quantities are the differential pressure error $\mathrm{E}$ and the error change rate EC. The output quantity is the opening degree state U. If setting the reference input differential pressure $\mathrm{Pr}$ to $0 \mathrm{~Pa}$, the value of the signal detected by the differential pressure sensor $\mathrm{P}$ can be equal to the differential pressure error $\mathrm{E}(\mathrm{E}=\mathrm{P}-\mathrm{Pr})$. In the method of 3.1, the error change rate EC is not taken into consideration. As a result, an overshoot or delay is likely to occur in the control system, causing the problem of discomfort or waste of the limited oxygen when the pilots breathe. Taking full account of those influences, we add the change rate in the pressure difference EC as an input of the system.

In the fuzzy controller, the electronic valves open in the case of negative pressure signal. The differential pressure sensor constantly sends the detected signal into the monitor controller. Then the controller adjusts the valves' opening degree $U$ and the opening time length. So the electronic valves can be closed in advance of the positive signal detected, which makes the oxygen supply equipment more efficient.

The fuzzy control rules of the opening degree of the electronic valves are as follows:

If $\mathrm{E}=\mathrm{Ai}$ and $\mathrm{EC}=\mathrm{Bi}$, then $\mathrm{U}$ is $\mathrm{Ci}$.

In the rules, $\mathrm{Ai}, \mathrm{Bi}, \mathrm{Ci}$ are the variable values of differential pressure error $\mathrm{E}$, differential pressure error change rate EC and electronic valves opening degree $U$, respectively. In the fuzzy control rules, the variable value of $\mathrm{E}$ contains four states, including "negative big" (NB), "negative medium" (NM), "negative small" (NS), "zero" (ZO); the variable value of EC contains three states, including " negative" (N), "zero" (ZO), "positive" (P); and the variable value of U contains four states, including "zero" (ZO), "big" (B), "medium" (M), "small" (S); the membership functions of E / EC / U are all triangular. That can be expressed as follows:

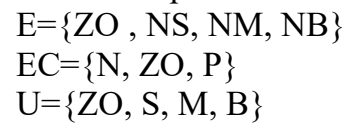

When EC is $\mathrm{N}$, the differential pressure error $\mathrm{E}$ continues to decrease, away from the setpoint" 0 "; When EC is $\mathrm{P}$, the differential pressure error $\mathrm{E}$ is increasing and approaching to 0 .

\subsection{Determination of fuzzy control rules}


1) If the differential pressure error $E$ is too large (NB) and the error change rate EC is negative $(\mathrm{N})$, the controller chooses a larger opening degree $\mathrm{U}(\mathrm{B})$ to meet the requirements of human breathing immediately; if the differential pressure error $\mathrm{E}$ is too large(NB) while the error change rate $\mathrm{EC}$ is positive $(\mathrm{P})$, the controller chooses a smaller opening degree $\mathrm{U}(\mathrm{M})$ or closed state to avoid the entering gas pass too quickly to cause discomfort;

2) If the differential pressure error $E$ is in medium (NM), the opening degree $U$ should have a reduction; if the error change rate $\mathrm{EC}$ is positive $(\mathrm{P}), \mathrm{U}$ should be reduced further.

3) When $E$ is small, $U$ is correspondingly reduced to ensure the respiratory stability, avoiding the phenomenon of overshoot. The controller adjusts the opening degree U according to the error change rate EC at the same time, so as not to apply the needed oxygen gas at the very beginning of the inhalation in time. The fuzzy control rules are shown in Table 2.

Table 2. Fuzzy control rules.

\begin{tabular}{|c|c|c|c|}
\hline \multirow{2}{*}{$\begin{array}{c}\text { differential pressure } \\
\text { error E }\end{array}$} & \multicolumn{3}{|c|}{ the error change rate EC } \\
\cline { 2 - 4 } & $\mathrm{N}$ & $\mathrm{ZO}$ & $\mathrm{P}$ \\
\hline ZO & $\mathrm{ZO}$ & $\mathrm{ZO}$ & $\mathrm{ZO}$ \\
\hline NS & $\mathrm{M}$ & $\mathrm{S}$ & $\mathrm{ZO}$ \\
\hline NM & $\mathrm{B}$ & $\mathrm{M}$ & $\mathrm{M}$ \\
\hline NB & $\mathrm{B}$ & $\mathrm{B}$ & $\mathrm{M}$ \\
\hline
\end{tabular}

The Mamdani fuzzy control is carried out by MATLAB tool in this paper. The model dealt by the tool of MATLAB has been shown in Fig. 4. The Mamdani fuzzy control is used to build the controller model and the center of gravity method is to solve the fuzzy model[6]. After solving the model, we obtain the control data of the opening degree in different states. When the $\mathrm{E}=-10.1 \mathrm{~Pa}, \mathrm{EC}=-$ $5.32 \mathrm{~Pa} / \mathrm{ms}$, the opening degree is $\mathrm{U}=\mathrm{S}$, only the electronic valve I opened. When the $\mathrm{E}=-18 \mathrm{~Pa}$, EC $=-12.9 \mathrm{~Pa} / \mathrm{ms}$, the opening degree is $\mathrm{U}=\mathrm{B}$, the double valves in the open state.

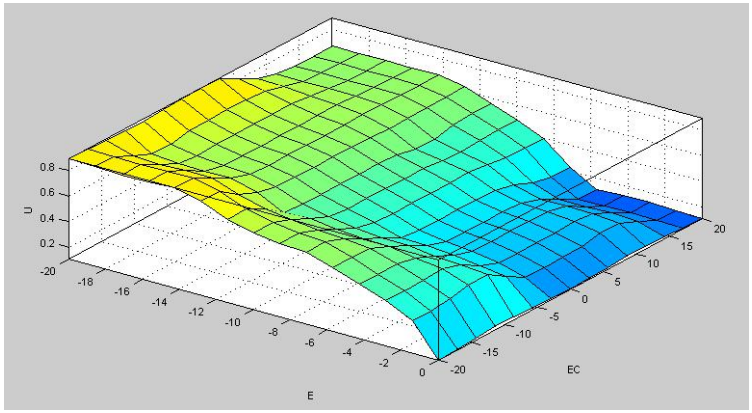

Fig. 4. The fuzzy control model.

In the practice, we store opening degree values calculated by MATLAB in the form of lookup table in the hardware. In real-time control, the E, EC data obtained by the acquisition, are sent to the monitor controller and the controller gains the opening degree value from the stored lookup table directly by some simple calculations.

\section{Results and analysis}

The pulse oxygen supply system introduced in this paper was tested in a cabin at low pressure with a fake lung which can simulate the human breathing in different frequencies under the condition of the ground lab. The oxygen air used in the experiment was the high pressure medical oxygen with the inlet tube pressure of $0.2 \mathrm{MPa}$. The test data are shown in Fig. 5. According to the control rules, the actual outlet tube pressure E should be controlled 
more than $-20 \mathrm{~Pa}$. The traditional single factor control belongs to the open loop control, and a delay or an overshoot of oxygen are extremely to happen to cause a discomfort. If the breathing state changing, such as a slight inspiration, the differential pressure changes are not obvious and quick enough to cause an in-time control action, then a delay occurs. A filling will occur with high-intensity breathing.

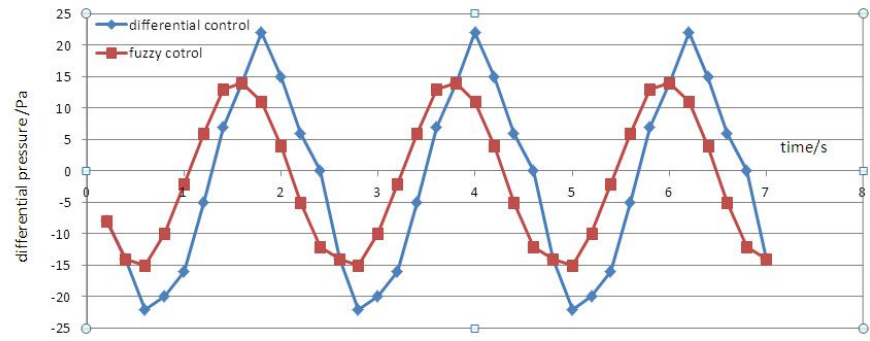

Fig. 5. The results curve of two control methods.

Compared to traditional differential control method, the fuzzy control method achieves the goals of adjusting the electronic valves opening degree from small to large switching in accommodate to the pace of breathing. The inspiration curve showed in Fig 5 suggests that the synchronization effect is better. There is no imbalance or overshoot caused by the lag and filling phenomenon.

\section{Concluding remarks}

The differential pressure error $\mathrm{E}$ and the error change rate $\mathrm{EC}$ are both considered to control the valves' opening degree, which avoids the shortcomings caused by the traditional open loop control method. The fuzzy control method in this paper will greatly improve the performance of the oxygen regulator with a practical debugging and further optimization, which could really provide a reliable and comfortable oxygen supply environment in the near future.

\section{References}

1. Xiao Huajun. Applied physiology of aviation oxygen protective equipment[M]. Beijing: Military Medical Science Press, 2005.

2. Zhu Xiaoji. Domestic research status in the respiratory physiology and protection about pilots in flight[J]. Practical Medicine Journal, 2007,24 (6): 746 -747.

3. Yu Xiao, Sun Bing and Lin Guiping. Atmegal28 Single-chip using on aircraft electronic oxygen regulator[J]. Microcomputer Applications, 2009, 30 (12): 50-56.

4. Sun Canfei, Cai Yuanyou and Long Haijun. Research on stepping motor fuzzy control technology applied in the aircraft electronic oxygen regulator[J]. Measurement and Control Technology, 2013,12 (4): 78-81.

5. Li Zuxin. Application of MATLAB in the design and simulation of fuzzy control system[J]. Journal of System Simulation, 2003, 15 (1): 132 -134.

6. Duan Yinghong and Yang Shuo. The fuzzy PID control of stepping motor[J]. Computer Simulation, 23.2 (2006): 290-293. 